Conclusions Here, we demonstrate that ACPA IgG, isolated from SF of RA patients, have the ability to enhance the RANKL-driven osteocalstogenesis from immature dendritic cells. Our findings suggest that ACPA might have a direct pathogenic effect in RA associated bone destruction.

\section{A8.3 DEFICIT OF S100A4 PREVENTS JOINT DESTRUCTION AND SYSTEMIC BONE LOSS IN hTNFtg MOUSE MODEL}

doi:10.1136/annrheumdis-2013-203222.3

\begin{abstract}
1.2 Michal Tomcik, ${ }^{2}$ Christina Böhm, ${ }^{2}$ Carina Scholtysek, 'Lucie Andres Cerezo, ${ }^{2}$ Wolfgang Baum, ${ }^{2}$ Clara Dees, ${ }^{2}$ Christian Beyer, ${ }^{2,3}$ Jerome Avouac, ${ }^{2}$ Pawel Zerr, ${ }^{2}$ Katrin Palumbo, ${ }^{2}$ Alfiya Distler, ${ }^{1}$ Radim Becvar, ${ }^{4}$ Oliver Distler, ${ }^{5}$ Mariam Grigorian, ${ }^{2}$ Gerhard Krönke, ${ }^{2}$ Georg Schett, ${ }^{2}$ Jörg HW Distler, 'Ladislav Senolt. 'Institute of Rheumatology and Department of Rheumatology, 1st Faculty of Medicine, Charles University, Prague, Czech Republic; ${ }^{2}$ Department of Internal Medicine III and Institute for Clinical Immunology, University of Erlangen-Nuremberg, Erlangen, Germany; ${ }^{3}$ Paris Descartes University, Rheumatology A Department, Cochin Hospital, Paris, France; ${ }^{4}$ Center of Experimental Rheumatology and Zurich Center of Integrative Human Physiology, University Hospital Zurich, Switzerland; 5 Institute of Cancer Biology, Danish Cancer Society, Copenhagen, Denmark
\end{abstract}

Background Our previous studies demonstrated increased levels of S100A4 protein in sera, synovial fluid and synovial membrane of patients with rheumatoid arthritis (RA) compared to osteoarthritis. S100A4 regulates apoptosis and induces production of matrix metalloproteinases by synovial fibroblasts. Furthermore, S100A4 stimulates synthesis of tumour necrosis factor (TNF)- $\alpha$ by mononuclear cells. The aim of this study was to investigate the effect of loss of S1004 in induction of experimental arthritis in the human TNF transgenic (hTNFtg) mouse model.

Methods We crossed the heterozygous hTNFtg mice with S100A4 knockout (S100A4 ${ }^{-/}$) mice. Mice were clinically assessed for paw swelling, grip strength and body weight weekly from 6 th to 14 th week of age in a blinded manner. Sections of hind paws and tibias were histologically analysed for synovial inflammation, cartilage loss, bone erosions, osteoclast numbers and bone formation parameters with the OsteoMeasure image analysis system.

Results In the group of hTNFtg; S100A4 $\%$ mice, paw swelling, grip strength and body weight were significantly improved compared to hTNFtg; S100A4 $4^{++}$( $p<0.01$ for all parameters). Consistent with the clinical observations, histological analysis of the tarsal joints of hTNFtg; S100A4 $\%$ mice showed reduced pannus formation (area of inflammation decreased by $66 \pm 3 \%, \mathrm{p}<0.01)$ and cartilage destruction (cartilage loss decreased by $63 \pm 6 \%, p<0.01$ ) compared to hTNFtg; S100A4 ${ }^{+/+}$mice. Similarly, osteoclast numbers were decreased by $84 \pm 3 \%(p<0.01)$ and bone erosions were less severe (area of bone erosion decreased by $81 \pm 4 \%, p<0.01$ ) in hTNFtg; $\mathrm{S} 100 \mathrm{~A} 4 \%$ mice. Furthermore, hTNFtg; S100A4 $\%$ mice were protected from systemic bone loss. Absence of S100A4 completely reversed increased osteoclast formation and bone resorption in hTNFtg mice. hTNFtg; S100A4\% mice had an increased bone volume per total volume $(\mathrm{BV} / \mathrm{TV})$ by $78 \pm 20 \%(\mathrm{p}<0.05)$ and a decrease in trabecular separation by $39 \pm 4 \%(p<0.05)$, decreased numbers of osteoclasts per bone perimeter ( $\mathrm{NOc} / \mathrm{BPm}$ decreased by $43 \pm 2 \%, \mathrm{p}<0.01$ ), decreased bone surface covered by osteoclasts (Oc.S/BS decreased by $52 \pm 3 \%, p<0.01$ ), increased numbers of osteoblasts per bone perimeter $(\mathrm{NOb} / \mathrm{BPm}$ increased by $129 \pm 20 \%, \mathrm{p}<0.05)$ and increased bone formation rate per bone surface (BFR/BS increased by $112 \pm$ $18 \%, \mathrm{p}<0.05)$ compared to hTNFtg; S100A4 $4^{+/+}$.

Conclusions These results suggest that inhibition of S100A4 effectively prevents induction of experimental arthritis via protecting against TNF-induced synovial inflammation, cartilage and bone destruction, and systemic bone loss. Our results support the role of S100A4 in the pathogenesis of RA where an increased S100A4 protein in circulation and locally at sites of inflammation may be linked to the process of aggressive fibroblast behaviour. Thus, S100A4 might represent a novel therapeutic target in RA.

\section{A8.4 FC-GLYCOSYLATION DETERMINES OSTEOCLASTOGENIC ACTIVITY OF IMMUUNE COMPLEXES}

doi:10.1136/annrheumdis-2013-203222.4

${ }^{1} \mathrm{U}$ Harre, ${ }^{1} \mathrm{R}$ Pfeifle, ${ }^{2} \mathrm{~A}$ Lux, ${ }^{3} \mathrm{~S}$ Frühbeißer, ${ }^{3} \mathrm{~F}$ Gröhn, ${ }^{1} \mathrm{G}$ Krönke, ${ }^{3} \mathrm{~F}$ Nimmerjahn, 'M Herrmann, 'G Schett. 'Department of Internal Medicine 3, Friedrich-Alexander University of Erlangen-Nuremberg, Germany; '2Department of Biology, FriedrichAlexander University of Erlangen-Nuremberg, Germany; ${ }^{3}$ Department of Chemistry and Pharmacy and Interdisciplinary Center for Molecular Materials, Friedrich-Alexander University of Erlangen-Nuremberg, Germany

Background and Objectives Autoantibodies recognising citrullinated proteins (ACPA) are highly specific for rheumatoid arthritis (RA), precede the clinical onset of the disease by years and are the strongest known risk factor for bone loss. We have recently shown that ACPA specific for citrullinated vimentin directly interact with osteoclast precursors and induce bone loss. In patients with RA, ACPA-containing immune complexes can be detected in synovial fluid and tissue. We hypothesised that (I) immune complexes directly promote osteoclast maturation and, consecutively, bone loss and that (II) the type of IgG-glycan is important for the interaction with osteoclast precursors, since ACPA have been shown to be hyposialylated.

Materials and Methods We differentiated preosteoclasts from human monocytes and stimulated them with artificial immune complexes generated by heat aggregation from pooled human IgC (IVIG). Part of the IgG had been pretreated with neuraminidase or PNGase F to remove sialic acid or the whole Fc glycan, respectively. For in vivo studies we injected murine immune complexes in the knee joints of C57-BL/6 mice.

Results Stimulation of preosteoclasts with immune complexes resulted in their dramatically increased maturation to osteoclasts. This effect was even more pronounced with complexes formed from desialylated IgG. Monomeric IgG and fully deglycosylated immune complexes did not alter osteoclast maturation. qPCR and FACS-analyses revealed that all $\mathrm{Fc} \gamma$ receptors $(\mathrm{Fc} \gamma \mathrm{R})$ are upregu-

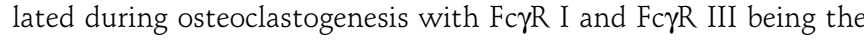
most prominent ones. Desialylated immune complexes induced the activation of spleen tyrosine kinase (Syk) and phospholipase $\mathrm{C} \gamma$ (PLC $\gamma$ ) as well as the upregulation of the transcription factor c-fos in preosteoclasts. Injection of murine immune complexes into the knee joints of C57-BL/6 mice caused accumulation of osteoclasts in the vicinity of the site of injection.

Conclusions Our data show that IgG immune complexes promote osteoclastogenesis. They upregulate the pro-osteoclastogenic transcription factor c-fos, after binding to activating Fc $\gamma$ Rs on preosteoclasts. This interaction is highly dependent on the absence of sialic acid in the Fc-glycan of the IgG. Altogether, we propose a novel mechanism by which ACPA promote bone loss independent of inflammation.

\section{A8.5 FIBROBLAST ACTIVATION PROTEIN ALPHA IN INFLAMMATORY BONE DESTRUCTION}

doi:10.1136/annrheumdis-2013-203222.5

Christina Wunrau, Corinna Wehmeyer, Marianne Heitzmann, Thomas Pap, Berno Dankbar. Institute of Experimental Musculoskeletal Medicine - IEMM, University Hospital Muenster, Muenster, Germany

Background The Fibroblast Activation Protein alpha (FAP $\alpha$ ) is an integral membrane serine protease that plays a major role in migration, wound healing, and metastasis. Based on recent studies that have implicated membrane-bound serine proteases in osteoclast migration, we studied the expression of FAP $\alpha$ in rheumatoid arthritis (RA) and analysed its role in osteoclast development under inflammatory conditions. 
Methods FAP $\alpha$ expression in vivo was determined by immunohistochemistry in human synovial tissues of patients with RA and osteoarthritis (OA) as well as in hind paws of tumour necrosis factor-alpha transgenic (hTNFtg) mice, which develop a RA-like destructive arthritis. In vitro expression and regulation of FAP $\alpha$ was analysed in differentiated osteoclasts cocultured with osteoblasts, RA or OA synovial fibroblasts (SF) by PCR. To scrutinise the role of FAP $\alpha$ in osteoclastogenesis, we analysed the in vitro differentiation of osteoclasts from wildtype (WT) and FAP $/$ mice (Oncology Research, Boehringer Ingelheim RCV, Vienna) by TRAP staining. In order to assess the role of FAP in arthritis severity, we crossed FAP/ and hTNFtg mice and performed TRAP staining.

Results RA synovial tissues demonstrated a high expression of FAP $\alpha$ throughout the tissue whereas in OA samples FAP $\alpha$ was expressed only in the lining layer. In vitro, no expression of FAP $\alpha$ was found in differentiated preosteoclasts and osteoclasts, but coculture experiments showed that RASF, but not OASF or osteoblasts, induce the expression of FAP $\alpha$ in preosteoclasts and osteoclasts. Consistent with the selective induction of FAP $\alpha$ in osteoclasts by RASF, FAP $\alpha$ expression was detected in osteoclasts at the invasion front of the hyperplastic synovial tissues in joints of hTNFtg mice. FAP /- mice show a severely diminished osteoclast formation compared to WT. We also found a lesser amount of osteoclasts in the hind paws of FAP/hTNFtg compared to hTNFtg.

Conclusions The disease-dependent expression of FAP $\alpha$ by osteoclasts in human RA and hTNFtg mice suggests an important role of FAP $\alpha$ in joint destruction in RA. The selective induction of FAP $\alpha$ in preosteoclasts and osteoclasts by RASF indicate that FAP $\alpha$ may be regulated through the interaction with the pannus tissue. The fact, that under inflammatory conditions the loss of FAP led to a reduced number of osteoclasts in the hind paws and FAP deficient bone marrow derived macrophages showed a reduced osteoclast formation, suggest a role of this serine protease in macrophage/osteoclast precursor migration and differentiation.

\section{A8.6 FRZB IS A CRITICAL MODULATOR OF CANONICAL WNT SIGNALLING IN CARTILAGE BIOLOGY}

doi:10.1136/annrheumdis-2013-203222.6

S Thysen, F Cailotto, FP Luyten, R Lories. Laboratory of Tissue Homeostasis and Disease, Skeletal Biology and Engineering Research Center, Department of Development and Regeneration, KU Leuven, Belgium

Background and Objectives Polymorphisms in Frizzled-related protein $(F R Z B)$, a WNT antagonist, have been associated with osteoarthritis $(\mathrm{OA})$. However, a recent meta-analysis failed to find a consistent effect of FRZB genetic variants on OA susceptibility. Our transcriptomics analysis in $F R Z B^{-/}$mice provided evidence for a tight regulation of WNT signalling and highlighted the complex role for $F R Z B$ in joint homeostasis. We previously demonstrated that $F R Z B^{-1}$ mice have increased damage when dramatically challenged by papain, collagenase or severe inflammation. As these models are acute and short-term, we aimed to further investigate the effect of $F R Z B$ loss in a true translational model of $O A$ and to study molecular interactions in the ATDC5 micromass in vitro model using RT-PCR and Western blot analysis.

Materials and Methods Surgical destabilisation of the medial meniscus (DMM) was performed on the right knee of eight-week-old male $F R Z B^{-/}$and wild-type mice. Eight weeks after surgery, mice were sacrificed and histological scores were determined for the femoral and tibial articular surfaces following the OARSI histopathology initiative guidelines.

Results Overexpression of FRZB in ATDC5 micro-masses boosted chondrogenesis with up-regulation of Col2a1 and Aggrecan transcription, whereas downregulation of $F R Z B$ lead to a decreased expression of Colza1 and Aggrecan. These results corresponded with a reduction or increase in the activation of canonical WNT signalling pathway, respectively. Fluctuating levels of $F R Z B$ did not influence the Wnt/CamKII signalling pathway. The semi-quantitative $O A R S I$ score showed a significant increase in cartilage erosion in DMM-operated $F R Z B^{-1}$ mice compared to wild-type.

Conclusions Our data show that, in addition to the higher susceptibility to $\mathrm{OA}$ in acute induced models, $F R Z B^{-/}$mice are more prone to $O A$ in a full translational model of the disease characterised by slowly progressive joint damage. Overexpression of $F R Z B$ stimulates chondrogenesis by its inhibitory role on Wnt/beta-catenin signalling.

\section{A8.7 FUNCTIONAL IMPAIRMENT IN AN ANIMAL MODEL FOR RHEUMATOID ARTHRITIS ASSESSED AS CHANGES IN GAIT IS DUE TO JOINT DESTRUCTION BUT NOT SYNOVIAL INFLAMMATION PER SE}

doi:10.1136/annrheumdis-2013-203222.7

Gregor Bauer, Martin Willburger, Constantin Aschauer, Aurica Jelinek, Tetyana Shvets, Birgit Niederreiter, Josef Smolen, Kurt Redlich, Silvia Hayer. Medical University of Vienna, Department of Internal Medicine III, Division of Rheumatology, 1090 Vienna, Austria

Objective To investigate the individual impact of synovial inflammation, subchondral bone erosion or cartilage damage on functional impairment in an animal model of Rheumatoid Arthritis (RA).

Methods We analysed gait profiles in human tumour necrosis factor transgene (hTNFtg) animals, using the video-based Catwalk gait analysis system (from Noldus, Netherlands). In this system, mice run along an illuminated glass plate. A digital camera measures light emissions resulting from the contact of paws on the glass plate. We evaluated gait profiles at different time points of disease (6, 10, 15 week of age) in hTNFtg animals. Wildtype littermates served as controls. Bodyweight and clinical signs of arthritis including paw swelling and grip strength were also evaluated. To investigate whether gait changes are pain related, we treated hTNFtg animals with diclofenac (50 mg/kg, i.p.) at week 10 and week 15 after birth and analysed gait profiles before, as well as $1 \mathrm{~h}$ and $3 \mathrm{~h}$ after treatment. To investigate reversibility in impaired gait profils, we treated hTNFtg mice with anti-TNF (Infliximab $10 \mathrm{mg} / \mathrm{kg}$ body weight, $2 \times$ per week) for 5 weeks starting 6 and 10 weeks after birth. To analyse inflammatory joint destruction, we quantitatively assessed the extend of synovial inflammation, subchondral bone erosion and cartilage damage on hematoxylin and eosine (H\&E), tartrate-resistant acid phosphatase (TRAP) and toluidine-blue stained paw sections. We performed correlation studies between gait parameters and the histopathological components as well as clinical signs.

Results We identified several gait parameters among them weight bearing, stride length and contact area of the paw to be significantly decreased in hTNFtg animals compared to sex- and age-matched wildtype animals. Moreover, we found a marked reduction in maximum intensity, an indicator for weight bearing, in week 10 and 15 compared to week 6 old hTNFtg mice. Similar effects were seen in print width, print area, print length, max contact max intensity and max contact area at different stages of disease. Interestingly, analgesic treatment with diclofenac $(50 \mathrm{mg} / \mathrm{kg}$, i.p. $)$, resulted in a better improvement of weight-bearing parameters in 10 week old hTNFtg mice than in 15 week old hTNFtg animals indicating an pain independent, irreversible functional impairment in progressed disease. To further investigate to which extend synovial inflammation, subchondral bone erosion or cartilage damage are responsible for the functional impairment of joints, we correlated these components with changes in different gait parameters. We observed strong correlations of various gait parameters with the amount of cartilage damage, whereas subchondral bone erosions correlated to a lesser extend and synovial inflammation did not correlate at all. 